
ИНСУЛИНОРЕЗИСТЕНТНОСТИ

\author{
(с Л.А. Руяткина ${ }^{1,2 *}$, Д.С. Руяткин ${ }^{1}$, И.С. Исхакова²
}

'ФГБОУ ВО «Новосибирский государственный медицинский университет» Министерства Здравоохранения РФ, Новосибирск, Россия

ГБУЗ Новосибирской области «Городская клиническая больница №1», Новосибирск, Россия

Высокая распространенность сахарного диабета 2 типа (СД2) и атеросклеротических сердечно-сосудистых заболеваний (СС3) определяет необходимость раннего выявлением и коррекции ключевых маркеров кардио-метаболического риска (KMP). Это профилактическое направление тесно связано с метаболическим синдромом (МС) на основе концепции инсулинорезистентности (ИР). Одновременно ИР является первым звеном патогенеза СД2 и признанным фактором риска атеротромбоза. Поэтому ранняя диагностика ИР имеет практическое значение как для выявления ранних нарушений углеводного обмена (НУО) и прогноза СД2, так и кардиологического риска.

Предложены альтернативные показатели для оценки ИР с включением липидных и антропометрических параметров, диагностическая и прогностическая значимость которых в плане КМР (НУО и ССЗ) оценена в рандомизированных клинических исследованиях в сравнении с индексом HOMA-IR и клэмп-тестом. Индекс ТуG (с расчётом на основе глюкозы плазмы и триглицеридов) согласуется с феноменом глюкозолипотоксичности с последующими метаболическими нарушениями в органах-мишенях. Предложены его производные: TyG-WC (TyG/окружность талии) и TYG-BMI (TyG/ ИMT). Применяют индексы LAP (показатель аккумуляции липидов) и VAI (индекс висцерального ожирения), а также TG/HDL (ТГ/ЛПВП). Выявлены их этнические и гендерные различия, сделаны попытки расчета отрезных точек для этих индексов.

КЛЮЧЕВЫЕ СЛОВА: метаболический синдром, инсулинорезистентность, кардио-метаболический риск, глюкозолипотоксичность, индексы инсулинорезистентности.

\title{
OPPORTUNITIES AND OPTIONS FOR SURROGATE ASSESSMENT OF INSULIN RESISTANCE
}

(c) Lyudmila A. Ruyatkina ${ }^{1,2 *}$, Dmitriy S. Ruyatkin², Irina S. Iskhakova ${ }^{2}$

${ }^{1}$ Novosibirsk State Medical University, Novosibirsk, Russia

${ }^{2}$ Novosibirsk Clinical Hospital №1, Novosibirsk, Russia

The high prevalence of type 2 diabetes mellitus (T2DM) and atherosclerotic cardiovascular diseases (CVD) determines the need for early detection and correction of key markers of cardio-metabolic risk (CMR). This prophylactic direction is closely related to metabolic syndrome (MS) based on the concept of insulin resistance (IR). At the same time, IR is the first link in the pathogenesis of T2DM and is a recognized risk factor for atherothrombosis. Therefore, early diagnosis of IR is of practical importance both for the detection of early disorders of carbohydrate metabolism (DCM) and prognosis of T2DM, and cardiological risk. Alternative indicators have been proposed for evaluating IR with the inclusion of lipid and anthropometric parameters, the diagnostic and prognostic significance of which in terms of CMR (DCM and CVD) has been evaluated in randomized clinical trials in comparison with the HOMA-IR index and clamp. The TyG index (calculated on the basis of plasma glucose and triglycerides) is consistent with the phenomenon of glucolipotoxicity with subsequent metabolic disorders in target organs. Its derivatives are proposed: TyG-WC (TyG / waist circumference) and TYG-BMI (TyG / BMI). Apply LAP indices (lipid accumulation index) and VAI (visceral obesity index), as well as TG / HDL (TG / HDL). Their ethnic and gender differences were revealed, attempts were made to calculate the cut-off points for these indices.

KEYWORDS: metabolic syndrome, insulin resistance, cardio-metabolic risk, glucolipotoxicity, insulin resistance indexes.

Профилактическое направление современной клинической медицины базируется на концепции метаболического синдрома (MC) [1], объединяющей метаболический и гормональный дисбаланс с гемодинамическими нарушениями. Подобный подход нацелен на раннее выявление и коррекцию ключевых маркеров кардиометаболического риска (КМР). Сделано много попыток на основании данных рандомизированных клинических исследований (РКИ), стартующих, по сути, от Фрамингемского исследования с разработкой по его результатам рискометра [2]. Создан ряд алгоритмов с идентификаци- ей основных факторов риска сердечно-сосудистых заболеваний (СС3): артериальной гипертензии (АГ), курения, сахарного диабета 2 типа (СД2), ожирения, семейной отягощенности, хронической болезни почек (ХБП) с учетом возрастных категорий [3].

В основе МС лежит инсулинорезистентность (ИР). G.M.Reaven (1988), представляя это понятие в своей Banting lecture «Role of insulin resistance in human disease», акцентировал внимание на ИР с компенсаторной гиперинсулинемией (ГИ) не только как основы нарушений углеводного обмена (НУО), но уже тогда показывал 
связь ИР с АГ и дислипидемией (ДЛП) [4]. R.A. DeFronzo и E.Ferrannini (1991) предположили, что ИР является синдромом, связанным с кластеризацией метаболических нарушений, в том числе инсулин-независимого СД, ожирения, АГ, липидных нарушений и атеросклеротических CC3 [5].

Позднее, резюмируя быстрое увеличение числа клинических синдромов и аномалий, связанных с ИР/ГИ (неалкогольную жировую болезнь печени, НЖБП; синдром поликистозных яичников; некоторые формы рака) G.M.Reaven предложил кластер этих изменений, связанных с дефектом в действии инсулина, отнести к термину «синдром инсулинорезистентности». Стало очевидно, что группа ИР/ ГИ объединяет много общепризнанных факторов риска СС3, среди которых ДЛП с акцентом на повышение триглицеридов (ТГ) и снижение холестерина липопротеидов высокой плотности (ХС-ЛПВП), повышенные концентрации в плазме крови ингибитора-1 активатора плазминогена (PAl-1), фибриногена, молекул адгезии, маркеров воспаления, диметиларгинина (эндогенного ингибитора фермента синтазы оксида азота) и гиперсимпатотонус [6]. Далее ИР определяют как наиболее распространенный фактор риска атеротромбоза [7]. Расширяется понятие ИР: кроме метаболической, эндокринной и неэндокринной, описаны её физиологические варианты [8].

Анализируя различные дефиниции МC [7]: WHO (1998), EGIR (1999), ATP (2005), IDF (2005) - кроме обязательно присутствующих диагностических признаков - АГ и ожирения, к основным маркерам относятся липидные нарушения (а именно, повышение ТГ и снижение ЛПВП), а также обсуждается ИР/ГИ. В этой связи G.M. Reaven, развивая концепцию, подчеркивает, что ИР и связанная с ней компенсаторная ГИ, являются независимыми предикторами трех клинических синдромов: ССЗ, СД2 и АГ; при этом именно наличие ИР определяет риск этих состояний у лиц с избыточной массой тела /ожирением. Поскольку этот риск неодинаков при ожирении, важно выявлять его самый высокий уровень [9], что обосновывает необходимость поиска методов оценки ИР, пригодных в реальной клинической практике.

Исследования последующихлет подтвердили роль ИР в тесной взаимосвязи метаболических и гемодинамических факторов: висцеральном ожирении, СД2, системном воспалении, ДЛП, нарушенном фибринолизе, дисфункции эндотелия (ДЭ) и атеросклерозе [10]. Безусловно, ИР служит первым звеном патогенеза СД2. При этом гипергликемия является отдаленным следствием ИР [11], при которой реализуется повреждающее действие ряда молекулярных метаболических нарушений, включая оксидативный и карбонильный стресс клеток-мишеней. Эндотелий сосудов не является классически инсулин-зависимым, но служит первичной мишенью инсулина. ДЭ сосудов сопряжена с СД2 и его сердечно-сосудистыми осложнениями [12]. Таким образом, ранняя диагностика ИР имеет большое практическое значение и в плане раннего прогноза НУО, и кардиологического риска.

В клинической практике наиболее простым и удобным методом оценки ИР служит измерение концентрации инсулина плазмы крови натощак [8]. В качестве суррогатного маркера ИР, значимо коррелирующим с клэмп-тестом, рассматривают т.н. гомеостатическую модель с расчётом по данным инсулинемии и гликемии натощак - индекс HOMA-IR; другой его вариант, HOMA-B, отражает функциональное состояние бета-клетки [13].

Индекс HOMA-IR широко использовался в качестве показателя ИР у взрослых, детей и подростков. Однако он отражает только печеночную ИР, учитывая способность базального инсулина подавлять глюконеогенез в печени в период голодания [14]. Разработана математическая модель HOMA2-IR, преимуществом которой перед предшественником является оценка периферийной ИР [15]. Однако уровни инсулина не стандартизированы в разных лабораториях, поскольку его нормальные значения крайне вариабельны $[8,14,16]$. Существует мнение, что использование различных индексов возможно в крупных эпидемиологических исследованиях и малоприменимо для индивидуальных измерений [12].

По результатам сравнительного с клэмп-тестом перекрестного исследования для оценки ИР предложен индекс TG/HDL-C (ТГ/XC-ЛПВП) [16] с попыткой определения отрезных точек (cut-off). Была показана способность индекса идентифицировать ИР-индивидуумов аналогично критериям ATP III (Adult Treatment Panel III) для диагностики МС (чувствительность 52\%, специфичность 85\%). Выявлена тесная ассоциация TG/HDL-C с ИP (по HOMA-IR), причем независимо от окружности талии (ОT) [17].

Результаты MESYAS study (Metabolic Syndrome in Active Subjects; $n=18$ 778) свидетельствуют, что показатели TG/HDL-C > 2,75 у мужчин и > 1,65 у женщин прогностически высоко значимы для развития MC, а также первого коронарного события независимо от индекса массы тела (ИМТ) [7]. Показана значимость TG/HDL-C в педиатрии в различных этнических популяциях: он оказался лучшим показателем, чем HOMA-IR при скрининге на MC у детей и подростков с ожирением [18]; этот индекс предложено использовать для идентификации детей с повышенным риском ИР-ассоциированной заболеваемости [19].

N.A. Iwani с соавт. (2017) обобщают данные по информативности индекса TG/HDL с акцентом на потребность в легко осуществимом, высоко точном и малозатратном диагностическом тесте для прогнозирования ИР. Так, TG/ HDL-C $\geq 3$ тесно коррелирует с резистентностью к инсулину у взрослых; является независимым фактором риска СС3 (ИБС) среди иранских мужчин; у детей наибольшие значения индекса отражают повышенный в 1.8-3.8 раза риск центрального ожирения, ИР, повышения СРБ, НЖБП, МС и увеличение толщины интима-медиа сонной артерии [20].

При этом информативность TG/HDL-C уточняется. По данным китайской популяции он служит предиктором ИР, но не функционального состояния $\beta$-клетки [21]. Наоборот, у женщин афроамериканок TG/HDL-C является слабым предиктором ИР, но обратная зависимость индекса с функцией $\beta$-клетки отражает его роль как простого инструмента эффективной идентификации женщин с риском СД2 в этой популяции [22]. Высказана мысль об этнических особенностях информативности TG/HDL-C [17].

При анализе проблем оценки КМР у детей и подростков с ожирением, D.Y.Үоо с соавт. (2017) акцентируют внимание на связи индекса TG/HDL-C не только с ИР и НУО, но также обсуждают его роль как маркера ДЭ и структурных сосудистых изменений в различных этнических популяциях [23]. Эпидемиологические исследования по- 
следних лет свидетельствуют о независимой ассоциации гипертриглицеридемии и липопротеидов, богатых ТГ (TRLS), с повышенным кардиоваскулярным риском [24]. Генетические исследования также подтверждают причинно-следственную связь ТГ и TRLs c CC3 [25].

Данные современных проспективных исследований подтверждают патогенетическую роль хронической ИР в развитии НУО. Так, Н. Malmström с соавт. (2018) в специальном анализе 296428 человек из когорты Swedish AMORIS study показали, что в случаях СД2 отмечались более высокие показатели ИМТ и уровни глюкозы плазмы натощак (ГПН) и ТГ по сравнению с контролем более, чем за 20 лет до постановки диагноза. То есть, диабетогенные процессы, связанные с ИР, действуют десятилетиями до развития СД [11]. При выборке из когорты AMORIS пациентов моложе 50 лет, оказалось, что около 50\% больших сердечно-сосудистых событий (ССС) в этом возрасте могут быть связаны с повышенным уровнем липидов и глюкозы крови [26].

В этой связи особое внимание привлекает концепция глюкозолипотоксичности, стартующей от представления о генерализованном характере феномена липотоксичности, необходимым условием для которой является гипергликемия в условиях ИР: глюкоза перестает быть источником энергии, что сопровождается активацией липолиза и повышением уровней свободных жирных кислот (СЖК) с потенциалом токсичности и метаболических нарушений в органах-мишенях - печени, сердце, сосудах, поджелудочной железе, мышцах [27]. То есть, хорошо известная взаимосвязь между ИР, уровнем инсулина и толерантностью к глюкозе в значительной степени опосредована изменениями концентрации СЖК [4]. Установлено, что отрицательные последствия глюкозолипотоксичности превышают сумму последствий изолированных глюкозо- и липотоксичности [28]. Этот феномен отражает тесную связь липидного и углеводного обменов.

С концепцией глюкозолипотоксичности в комплексе с данными AMORIS [11] согласуется другой индекс, предложенный для суррогатной характеристики ИP - ТуG (логарифмическое соотношениеуровней ТГиГПН), показав аналогичную корреляцию с ИР, измеренную с помощью клэмп-теста, независимо от пола, наличия ожирения и СД [29] и классической моделью НОМА-IR для оценки ИР [30, 31].

Проспективные клинические исследования позволяют уточнить диагностическую и прогностическую информативность данного индекса. По итогам Vascular-Metabolic CUN cohort ( $n=4820$, длительность наблюдения 8.84 4 4.39 лет) показано, что индекс TyG, является лучшим предиктором потенциального развития СД2 у пациентов с нормогликемией, чем ГПН или ТГ [32]. J.W.Lee с соавт. (2018) на основании данных KOGES (Korean Genome and Epidemiology Study: $n=7708$ в возрасте 40-69 лет) считают, что индекс ТуG следует рассматривать как инструмент скрининга для выявления людей с высоким риском развития СД2. При этом площадь под кривой (AUC) для TyG была больше, чем у HOMA-IR; отрезную точку для TyG определили как 4.69 (чувствительность 62.1\%; специфичность 63.1\%) [33].

J. Salazar с соавт. (2018) на основании результатов Маracaibo City Metabolic Syndrome Prevalence Study ( $n=2004$ лиц старше 18 лет) считают TyG пригодным для иденти- фикации ИР, предлагая схожую по значениям cut-off: 4.49 с более высокой чувствительностью и специфичностью (82.6\% и 82.1\% соответственно). При этом авторы считают, что необходимо уточнить прогностическую ценность индекса TyG не только для СД2, но и сердечно-сосудистого риска [34]. Shin K.-А. (2017), рекомендуя индекс ТуG как маркер ИР в идентификации риска МС, обосновывает с этой целью отрезную точку 8.81 с высокой чувствительностью и специфичностью (86.7\% и $80.1 \%$ соответственно) [35].

При анализе значений отрезных точек, предложенных разными авторами, обращает внимание их практически двукратное отличие: 4.69 [33] и 4.49 [34] против 8.81 [35]. Это различие объяснимо использованием двух модификаций формулы для вычисления индекса: ТуG = Ln [TG (мг / дл) × FPG (мг / дл) / 2] и TyG = Ln [TG (мг / дл) $\times$ FPG (мг / дл)] 2, значения по которым отличаются в 2 раза. С учетом этого факта важно, что показатели точек cut-off, полученные на разных популяциях, очень близки.

E.K. Hameed (2019) на основе анализа исследований в Бразилии и Мексике считает, что индекс TyG, хорошо коррелируя с данными клэмпа, является многообещающим суррогатным маркером для оценки ИР. Он также может быть использован для выявления метаболически активного ожирения у лиц с нормальной массой тела и как предиктор субклинического атеросклероза, верифицированного по кальцификации коронарных артерий с помощью компьютерной томографии и НЖБП [36].

Предложены производные индекса ТуG с включением антропометрических параметров: TyG-WC (произведение TyG и окружности талии, ОT) и TYG-BMI (произведение TyG и ИMT). Это комплексное направление оценки ИР продолжают индексы на основе липидных и антропометрических показателей: LAP (lipid accumulation product) показатель аккумуляции липидов (формула для мужчин: (ОТ (см) - 65) умножить на ТГ (ммоль/л); для женщин (ОT - 58) х ТГ) [37] и VAI [30]- индекс висцерального ожирения (формула для мужчин: [ОТ/(39.68 + $1.88 \times$ ИМТ)] $\times$

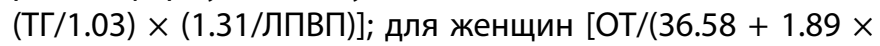
ИМТ)] $\times($ ТГ/0.81) × (1.52/ЛПВП)], где значения ТГ и ЛПВП в ммоль/л)]. На основании когортного проспективного исследования в Корее $(n=1544$ родственников первой линии пациентов с СД2) индекс TyG-WC проявил себя как клинически эффективный маркер для раннего выявления рисков предиабета и диабета в данной когорте лиц [38].

Представляет интерес сравнение различных индексов для оценки ИР между собой. Так, в рамках China Health and Nutrition Survey 2009 индексы TG/HDL-C, VAl и TyG тесно коррелировали с ИР, оцененной по HOMA-IR [30]. В исследовании L.K.Er с соавт. (2016) среди 511 тайваньцев индекс TG/HDL-C был более эффективным детектором ИР (оцененной по HOMA-IR) в сравнении с другими липидными переменными или соотношениями: VAl, TyG и TyG-BMI [39].

В то же время в китайском когортном исследовании сельского населения $(\mathrm{n}=11113$ лиц, медиана наблюдения 6.0 лет) прогнозирование СД2 не улучшалось при использовании индексов TyG, VAI и LAP по сравнению только с ГПН и ОТ [40]. Результаты Tehran Lipid and Glucose Study ( $n=4419$, медиана наблюдения 12.0 лет) свидетельствуют, что ГПН является более сильным предик- 
тором СД2, чем индексы TyG, TG/HDL-C и HOMA-IR, хотя TyG-индекс был лучше, чем TG/HDL-C у обоих полов [41]. M. Zhou с соавт. (2016) уточняют, что в китайской популяции с различной толерантности к глюкозе TG/HDL-C и ТГ могут быть предикторами ИР, но не факторами, определяющими функцию $\beta$-клеток [21]. Эти данные согласуются с исследованием T.Du с соавт. (2014), в котором индексы TG/HDL-C, VAI и TyG оказались лучшими маркерами для ранней идентификации ИP [30]. J. W.Lee с соавт. (2018) считают, что индекс TyG в сравнении с ГПН является более надежным предиктором СД2 [33].

Основываясь на информативности и доступности TyG, оценен потенциал использования индекса и его производных, TyG-WC и TYG-BMI, для оценки гликемического контроля в выборке иракских пациентов с СД2. Корреляции ТуG как с HbA1c, так и с ИР, оцененной по HOMA-IR, были сильнее в сравнении с производными TyG, что позволяет расценивать его как полезный и доступный инструмент для оценки долгосрочного контроля гликемии у пациентов с СД2 [35].

Одновременно индексы TyG и TG/HDL-C показывают большие возможности по сравнению с HOMA2-IR в прогнозировании коронарного риска, что демонстрирует значимость триглицеридемии в патофизиологии ИР. При этом J.Salazar с соавт. (2018) считают, что большая прогностическая способность индексов, имеющих показатель TG сыворотки в расчетах, не зависит от их способности отражать ИР в скелетных мышцах или жировой ткани [42].

Кроме того, существуют гендерные особенности в ИР и её потенциальном влиянии на сердечно-сосудистый риск; более высокая прогнозирующая способность коронарного риска у женщин, возможно, связана с гендерными различиями в уровнях ТГ и ХС-ЛПВП [43].

Показатели ТГ также входят в формулы вычисления индексов VAI и LAP, которые, по мнению Mohsen $M$ et al (2018), являются хорошими суррогатными маркерами абдоминального ожирения [44]. Смысловое значение этих индексов отражает, по сути, вероятность ускорения/усугубления ИБС при сочетании ОТ с высоким уровнем ТГ и СЖК. R.F.Yang с соавт. (2018) показали, что пациенты с высокими уровнями ТГ и показателями ОТ более подвержены коронарному атеросклерозу, а наличие обоих этих факторов у пациентов с ИБС сопровождается более высокими уровнями СЖК и более выраженным воспалением [45]. При этом уровни СЖК у лиц с комбинацией высоких параметров ТГ и ОТ были значительно выше, чем просто при наличии повышения ТГ.

Выявлена связь TG/HDL-C и показателя жесткости сосудистой стенки, оцененной по скорости распростра- нения пульсовой волны при измерении плече-лодыжечного индекса (baPWV = brachial-ankle pulse wave velocity), однако эти отношения нелинейны: TG/HDL-C положительно коррелировал c baPWV при значениях меньше 5.6 [46]. На основании проспективного исследования ( $n=14673$, средний период наблюдения 6.4 года) аргументируется возможность показателя baPWV в повышении эффективности прогнозирования риска развития СС3 по сравнению с оценкой по Фрамингему, которая основана на традиционных факторах риска сердечно-сосудистых заболеваний [47].

По результатам когортного исследования C.Chi c coавт. (2018) привлекают внимание к тесным взаимосвязям с повреждением органов-мишеней (включая жесткость аорты - aortic stiffness, заболевания периферических артерий и ХБП и считая их определяющим фактором КМР) именно комбинированных липидных индексов в отличии от отдельных липидных показателей [48]. Результаты перекрестного исследования $(n=17596)$ подтверждают высокую корреляцию TG/HDL-C с факторами KMP и субклиническим повреждением органов-мишеней. Более того, индекс TG/HDL-C реагировал на различные кардио-метаболические факторы риска даже при референсных показателях ТГ и ХС-ЛПВП [49]. В качестве отрезных точек в данной китайской популяции предложено использовать значения TG/HDL-C $\geq 1.255$ у мужчин и $\geq 0.865$ у женщин.

Таким образом, существуют расовые и этнические различия KMP [50]. Поэтому в группах высокого риска должны применяться индивидуальные стратегии профилактики и лечения, основанные на все еще ограниченных данных проспективных клинических исследованиях [51].

Итак, концепция инсулинорезистентности в современном понимании не является «привилегией» сугубо диабетологии. Подход к верификации ИР с включением липидных параметров отражает более широкий взгляд на ключевую современную патологию с высоким КМР, лидирующую в плане заболеваемости и смертности, в патогенезе которой лежит синдром инсулинорезистентности. Важно, что во всех формулах индексов, наиболее тесно коррелирующих с HOMA-IR, присутствует показатель триглицеридов. Согласуясь с результатами проспективных исследований в отношении прогностической оценки КМР, наиболее привлекательными по чувствительности и специфичности являются индексы TyG и TG/HDL-C. Они могут быть полезным инструментом для выявления людей с высоким риском целого ряда расстройств и последующего раннего вмешательства. Таким образом, данные расширяют клиническую полезность этих индексов за пределами выявления пациентов с ИР.

\section{СПИСОК ЛИТЕРАТУРЫ | REFERENCES}

1. Cabré J-J, Martín F, Costa B, et al. Metabolic Syndrome as a Cardiovascular Disease Risk Factor: Patients Evaluated in Primary Care. BMC Public Health. 2008;8(1):251. doi: 10.1186/1471-2458-8-251

2. Wilson PWF, D'Agostino RB, Levy D, et al. Prediction of Coronary Heart Disease Using Risk Factor Categories. Circulation. 1998:97(18):1837-1847. doi: 10.1161/01.CIR.97.18.1837

3. Piepoli MF, Hoes AW, Agewall S, et al. 2016 European Guidelines on cardiovascular disease prevention in clinical practice. Eur Heart J. 2016;37(29):2315-2381. doi: 10.1093/eurheartj/ehw106
4. Reaven GM. Role of Insulin Resistance in Human Disease. Diabetes. 1988;37(12):1595-1607. doi: 10.2337/diab.37.12.1595

5. DeFronzo RA, Ferrannini E. Insulin Resistance: A Multifaceted Syndrome Responsible for NIDDM, Obesity, Hypertension, Dyslipidemia, and Atherosclerotic Cardiovascular Disease. Diabetes Care. 1991;14(3):173-194. doi: 10.2337/diacare.14.3.173

6. Reaven GM. Insulin resistance, the insulin resistance syndrome, and cardiovascular disease. Panminerva Med. 2005;47(4):201-210. PMID: 16489319 
7. Cordero A, Alegria-Ezquerra E.TG/HDL ratio as surrogate marker for insulin resistance. E-Journal of Cardiology Practice [Internet]. 2009;8(16) [cited 2019 Mar 16] Available from: https://www.escardio. org/Journals/E-Journal-of-Cardiology-Practice/Nolume-8/TG-HDL-ratio-as-surrogate-marker-for-insulin-resistance

8. Майоров А.Ю., Урбанова К.А., Галстян Г.Р. Методы количественной оценки инсулинорезистентности // Ожирение и метаболизм. - 2009. - T.6. - №2. - C.19-23. [Mayorov AY, Urbanova KA, Galstyan GR, et al. Methods for quantificative assessment of insulin resistance. Ožirenie i metabolizm. 2009;6(2):19-23 (In Russ.)] doi: 10.14341/2071-8713-5313

9. Reaven GM. Insulin Resistance: the Link Between Obesity and Cardiovascular Disease. Med Clin North Am. 2011;95(5):875-892. doi: 10.1016/j.mcna.2011.06.002

10. Conde SV, Ribeiro MJ, Melo BF, et al. Insulin resistance: a new consequence of altered carotid body chemoreflex? J Physiol. 2017;595(1):31-41. doi: 10.1113/JP271684

11. Malmström H, Walldius $\mathrm{G}$, Carlsson $\mathrm{S}$, et al. Elevations of metabolic risk factors 20 years or more before diagnosis of type 2 diabetes: Experience from the AMORIS study. Diabetes, Obes Metab. 2018;20(6):1419-1426. doi: 10.1111/dom.13241

12. Дедов И.И., Ткачук В.А., Гусев Н.Б., и др. Сахарный диабет 2 типа и метаболический синдром: молекулярные механизмы, ключевые сигнальные пути и определение биомишеней для новых лекарственных средств // Сахарный диабет. - 2018. - Т.21. - №5. C.364-375. [Dedov II, Tkachuk VA, Gusev NB, et al. Type 2 diabetes and metabolic syndrome: identifi cation of the molecular mechanisms, key signaling pathways and transcription factors aimed to reveal new therapeutical targets. Diabetes Mellitus. 2018;21(5):364-375. (In Russ.)] doi: 10.14341/DM9730

13. Festa A, Williams K, Hanley AJG, Haffner SM. $\beta$-Cell Dysfunction in Subjects With Impaired Glucose Tolerance and Early Type 2 Diabetes: Comparison of Surrogate Markers With First-Phase Insulin Secretion From an Intravenous Glucose Tolerance Test. Diabetes. 2008:57(6):1638-1644. doi: 10.2337/db07-0954

14. Irace C, Carallo C, Scavelli FB, et al. Markers of insulin resistance and carotid atherosclerosis. A comparison of the homeostasis model assessment and triglyceride glucose index. Int J Clin Pract. 2013;67(7):665-672. doi: 10.1111/ijcp.12124

15. Wallace TM, Levy JC, Matthews DR. Use and Abuse of HOMA Modeling Diabetes Care. 2004;27(6):1487-1495. doi: 10.2337/diacare.27.6.1487

16. McLaughlin T, Abbasi F, Cheal K, et al. Use of Metabolic Markers To Identify Overweight Individuals Who Are Insulin Resistant. Ann Intern Med. 2003;139(10):802-809. doi: 10.7326/0003-4819-139-10-200311180-00007

17. Ren $X$, Chen $Z$ a., Zheng $S$, et al. Association between Triglyceride to HDL-C Ratio (TG/HDL-C) and Insulin Resistance in Chinese Patients with Newly Diagnosed Type 2 Diabetes Mellitus. PLoS One. 2016;1 (4):e0154345. doi: 10.1371/journal.pone.0154345

18. Liang J, Fu J, Jiang Y, et al. TriGlycerides and high-density lipoprotein cholesterol ratio compared with homeostasis model assessment insulin resistance indexes in screening for metabolic syndrome in the chinese obese children: a cross section study. BMC Pediatr. 2015;15(1):138. doi: 10.1186/s12887-015-0456-y

19. Giannini C, Santoro N, Caprio S, et al. The Triglyceride-to-HDL Cholesterol Ratio. Diabetes Care. 2011;34(8):1869-1874. doi: 10.2337/dc10-2234

20. Iwani NAKZ, Jalaludin MY, Zin RMWM, et al. Triglyceride to HDL-C Ratio is Associated with Insulin Resistance in Overweight and Obese Children. Sci Rep. 2017;7(1):40055. doi: 10.1038/srep40055

21. Zhou M, Zhu L, Cui X, et al. The triglyceride to high-density lipoprotein cholesterol (TG/HDL-C) ratio as a predictor of insulin resistance but not of $\beta$ cell function in a Chinese population with different glucose tolerance status. Lipids Health Dis. 2016;15(1):104. doi: 10.1186/s12944-016-0270-z

22. Maturu A, DeWitt P, Kern PA, Rasouli N. The triglyceride to high-density lipoprotein cholesterol (TG/HDL-C) ratio as a predictor of $\beta$-cell function in African American women. Metabolism. 2015;64(5):561-565. doi: 10.1016/j.metabol.2015.01.004

23. Yoo D-Y, Kang YS, Kwon EB, Yoo E-G. The triglyceride-to-high density lipoprotein cholesterol ratio in overweight Korean children and adolescents. Ye J, ed. Ann Pediatr Endocrinol Metab. 2017;22(3):158-163. doi: 10.6065/apem.2017.22.3.158

24. Toth P. Triglyceride-rich lipoproteins as a causal factor for cardiovascular disease. Vasc Health Risk Manag. 2016;2016(12):171-183. doi: 10.2147/NHRM.S104369
25. Budoff M. Triglycerides and Triglyceride-Rich Lipoproteins in the Causal Pathway of Cardiovascular Disease. Am J Cardiol. 2016;118(1):138-145. doi: 10.1016/j.amjcard.2016.04.004

26. Ivert T, Malmström H, Hammar N, et al. Cardiovascular events in patients under age fifty with early findings of elevated lipid and glucose levels - The AMORIS study. Vinciguerra M, ed. PLoS One. 2018;13(8):e0201972. doi: 10.1371/journal.pone.0201972

27. Руяткина Л.А., Руяткин Д.С., Землянухина С.А. «Болевые» точки диабетических ангиопатий: фокус на гипертриглицеридемию и возможности фенофибрата // Фарматека. - 2016. - №5(318). - С.14-21. [Rujatkina LA, Rujatkin DS, Zemljanuhina SA. Diabetic angiopathy "painful" points: focus on hypertriglyceridemia and fenofibrate potential. Farmateka. 2016;(5):14-21 (In Russ.)]

28. Аметов А.С., Камынина Л.А., Ахмедова 3.А. Глюкозо- и липотоксичность - взаимоотягощающие факторы при сочетании сахарного диабета типа 2 и ожирения // Эндокринология: новости, мнения, обучение. - 2014. - T.4. - №4. - C.20-23. [Ametov A.S., Kamynina L.A., Akhmedova Z.A. Glyukozo- i lipotoksichnost' - vzaimootyagoshchayushchie faktory pri sochetanii sakharnogo diabeta tipa $2 \mathrm{i}$ ozhireniya. Ėndokrinologiâ. Novosti, mneniâ, obučenie. 2014;(4):20-23 (In Russ.)]

29. Guerrero-Romero F, Simental-Mendía LE, González-Ortiz M, et al. The Product of Triglycerides and Glucose, a Simple Measure of Insulin Sensitivity. Comparison with the Euglycemic-Hyperinsulinemic Clamp. J Clin Endocrinol Metab. 2010;95(7):3347-3351. doi: 10.1210/jc.2010-0288

30. DuT, Yuan G, Zhang M, et al. Clinical usefulness of lipid ratios, visceral adiposity indicators, and the triglycerides and glucose index as risk markers of insulin resistance. Cardiovasc Diabetol. 2014;13(1):146. doi: 10.1186/s12933-014-0146-3

31. Simental-Mendía LE, Rodríguez-Morán M, Guerrero-Romero F. The Product of Fasting Glucose and Triglycerides As Surrogate for Identifying Insulin Resistance in Apparently Healthy Subjects. Metab Syndr Relat Disord. 2008;6(4):299-304. doi: 10.1089/met.2008.0034

32. Navarro-González D, Sánchez-Iñigo L, Pastrana-Delgado J, Fernández-Montero A, Martinez JA. Triglyceride-glucose index (TyG index) in comparison with fasting plasma glucose improved diabetes prediction in patients with normal fasting glucose: The Vascular-Metabolic CUN cohort. Prev Med (Baltim). 2016;86:99-105. doi: 10.1016/j.ypmed.2016.01.022

33. Lee J-W, Lim N-K, Park H-Y. The product of fasting plasma glucose and triglycerides improves risk prediction of type 2 diabetes in middle-aged Koreans. BMC Endocr Disord. 2018;18(1):33. doi: 10.1186/s12902-018-0259-x

34. Salazar J, Bermúdez V, Calvo M, et al. Optimal cutoff for the evaluation of insulin resistance through triglyceride-glucose index: A cross-sectional study in a Venezuelan population. F1000Research. 2018;6:1337. doi: 10.12688/f1000research.12170.3

35. Shin K-A. Triglyceride and Glucose (TyG) Index is a Clinical Surrogate Marker for the Diagnosis of Metabolic Syndrome. Ye J, ed. Biomed SCi Lett. 2017;23(4):348-354. doi: 10.15616/BSL.2017.23.4.348

36. Hameed EK. TyG index a promising biomarker for glycemic control in type 2 Diabetes Mellitus. Diabetes Metab Syndr Clin Res Rev. 2019;13(1):560-563. doi: 10.1016/j.dsx.2018.11.030

37. Kim B, Choi HY, Kim W, et al. The cut-off values of surrogate measures for insulin resistance in the Korean population according to the Korean Genome and Epidemiology Study (KOGES). PLoS One. 2018;13(11):e0206994. doi: 10.1371/journal.pone.0206994

38. Zheng S, Shi S, Ren X, et al. Triglyceride glucose-waist circumference, a novel and effective predictor of diabetes in first-degree relatives of type 2 diabetes patients: cross-sectional and prospective cohort study. J Trans/ Med. 2016;14(1):260. doi: 10.1186/s12967-016-1020-8

39. Er L-K, Wu S, Chou H-H, et al. Triglyceride Glucose-Body Mass Index Is a Simple and Clinically Useful Surrogate Marker for Insulin Resistance in Nondiabetic Individuals. PLoS One. 2016;11(3):e0149731. doi: 10.1371/journal.pone.0149731

40. Wang $B$, Zhang $M$, Liu Y, et al. Utility of three novel insulin resistance-related lipid indices for predicting type 2 diabetes mellitus among people with normal fasting glucose in rural China. J Diabetes. 2018;10(8):641-652. doi: 10.1111/1753-0407.12642

41. Tohidi M, Baghbani-Oskouei A, Ahanchi NS, et al. Fasting plasma glucose is a stronger predictor of diabetes than triglyceride-glucose index, triglycerides/high-density lipoprotein cholesterol, and homeostasis model assessment of insulin resistance: Tehran Lipid and Glucose Study. Acta Diabetol. 2018;55(10):1067-1074. doi: 10.1007/s00592-018-1195-y 
42. Salazar J, Bermúdez V, Olivar LC, et al. Insulin resistance indices and coronary risk in adults from Maracaibo city, Venezuela: A cross sectional study. F1000Research. 2018;7:44. doi: 10.12688/f1000research.13610.1

43. Kim SH, Reaven G. Sex Differences in Insulin Resistance and Cardiovascular Disease Risk. J Clin Endocrinol Metab. 2013;98(11):E1716-E1721. doi: 10.1210/jc.2013-1166

44. Mazidi M, Gao H, Kengne AP. Lipid accumulation product and visceral adiposity index are associated with dietary patterns in adult Americans. Medicine (Baltimore). 2018;97(19):e0322. doi: 10.1097/MD.0000000000010322

45. Yang R-F, Zhang H, Wang Z, Liu X-Y, Lin Z. A study on the relationship between waist phenotype, hypertriglyceridemia, coronary artery lesions and serum free fatty acids in adult and elderly patients with coronary diseases. Immun Ageing. 2018;15(1):14. doi: 10.1186/s12979-018-0119-6

46. Chen C, Dai J-L. Triglyceride to high-density lipoprotein cholesterol (HDL-C) ratio and arterial stiffness in Japanese population: a secondary analysis based on a cross-sectional study. Lipids Health Dis. 2018;17(1):130. doi: 10.1186/s12944-018-0776-7
47. Ohkuma T, Ninomiya T, Tomiyama H, et al. Brachial-Ankle Pulse Wave Velocity and the Risk Prediction of Cardiovascular Disease. Hypertension. 2017;69(6):1045-1052. doi: 10.1161/HYPERTENSIONAHA.117.09097

48. Chi C, Teliewubai J, Lu Y-Y, et al. Comparison of various lipid parameters in association of target organ damage: a cohort study. Lipids Health Dis. 2018;17(1):199. doi: 10.1186/s12944-018-0800-y

49. Cao X, Wang D, Zhou J, Chen Z. Comparison of lipoprotein derived indices for evaluating cardio-metabolic risk factors and subclinical organ damage in middle-aged Chinese adults. Clin Chim Acta. 2017;475:22-27. doi: 10.1016/j.cca.2017.09.014

50. Osei K, Gaillard T. Disparities in Cardiovascular Disease and Type 2 Diabetes Risk Factors in Blacks and Whites: Dissecting Racial Paradox of Metabolic Syndrome. Front Endocrinol (Lausanne). 2017;8:204. doi: 10.3389/fendo.2017.00204

51. Dal Canto E, Farukh B, Faconti L. Why are there ethnic differences in cardio-metabolic risk factors and cardiovascular diseases? JRSM Cardiovasc Dis. 2018;7:204800401881892. doi: $10.1177 / 2048004018818923$

\section{ИНФОРМАЦИЯ ОБ АВТОРАХ [AUTHORS INFO]}

*Руяткина Людмила Александровна, д.м.н., профессор [Lyudmila A. Ruyatkina, ScD, professor];

ORCID: https://orcid.org/0000-0002-6762-5238; eLibrary SPIN: 1895-7664; e-mail: larut@list.ru

Руяткин Дмитрий Сергеевич, к.м.н. [Dmitriy S. Ruyatkin, PhD]; ORCID: https://orcid.org/0000-0003-3431-5943; eLibrary SPIN: 5433-3153; e-mail: dr79@mail.ru

Исхакова Ирина Сергеевна, к.м.н. [Irina S. Iskhakova, PhD]; e-mail: tyutyunjon@mail.ru

\section{ЦИТИРОВАТЬ:}

Руяткина Л.А., Руяткин Д.С., Исхакова И.С. Возможности и варианты суррогатной оценки инсулинорезистентности // Ожирение и метаболизм. — 2019. — Т.16. — №1. — С. 27-32. doi: 10.14341/omet10082

\section{TO CITE THIS ARTICLE:}

Ruyatkina LA, Ruyatkin DS, Iskhakova IS. Opportunities and options for surrogate assessment of insulin resistance. Obesity and metabolism. 2019;16(1):27-32. doi: 10.14341/omet10082 